\title{
Neoadjuvant therapy for pancreatic cancer
}

\author{
Jean Gugenheim ${ }^{1,2}\left[\right.$. Anna Crovetto ${ }^{3} \cdot$ Niccolo Petrucciani $^{3}$
}

Received: 5 May 2021 / Accepted: 30 September 2021 / Published online: 9 October 2021

(C) Italian Society of Surgery (SIC) 2021

\begin{abstract}
Multimodal treatment including surgery and chemotherapy is considered the gold standard treatment of pancreatic cancer by most guidelines. Neoadjuvant therapy (NAT) has been seen as a possible treatment option for resectable, borderline resectable and locally advanced $\mathrm{PaC}$. The aim of this paper is to offer a state-of-the-art review on neoadjuvant treatments in the setting of pancreatic ductal adenocarcinoma. A systematic literature search was performed using PubMed, Cochrane, Web of Science and Embase databases, in order to identify relevant studies published up to and including July 2021 that reported and analyzed the role of neoadjuvant therapy in the setting of pancreatic carcinoma. Most authors are concordant on the strong role of neoadjuvant therapy in the setting of borderline resectable pancreatic cancers. Recent randomized trials demonstrated improvement of R0 rate and survival after NAT in this setting. Patients with locally advanced cancers may become resectable after NAT, with better results than those obtained with palliative therapies. Even in the setting of resectable cancers, NAT is being evaluated by ongoing randomized trials. Chemotherapy regimens in the setting of NAT and response to NAT are discussed. NAT has an important role in the multimodal treatment of patients with borderline resectable pancreatic cancer. It has a role in patients with locally advanced tumors as it can allow surgical resection in a relevant proportion of patients. For resectable pancreatic cancers, the role of NAT is under evaluation by several randomized trials.
\end{abstract}

Keywords Pancreatic cancer $\cdot$ Neoadjuvant therapy $\cdot$ Surgery $\cdot$ Chemotherapy $\cdot$ Pancreas

\section{Introduction}

Ductal adenocarcinoma of the pancreas $(\mathrm{PaC})$ is one of the most aggressive types of cancer, with an overall 5-year survival around 9\%. More than 57,000 new cases are expected in 2020 in the United States, causing more than 47,000 estimated deaths [1]. Its incidence has risen in the last years, and by $2030 \mathrm{PaC}$ is expected to represent one of the leading causes of cancer-related deaths [2,3]. The only curative option for $\mathrm{PaC}$ is surgical resection, but only a minority of patients can be considered eligible for surgery,

Jean Gugenheim gugenheim.j@chu-nice.fr

1 Université Côte d'Azur, Nice, France

2 Division of Digestive Surgery and Liver Transplantation, Archet 2 Hospital, University Hospital of Nice, 151 Route de Saint-Antoine, 06200 Nice, France

3 Faculty of Medicine and Psychology, Department of Medical and Surgical Sciences and Translational Medicine, Sant'Andrea Hospital, Sapienza University of Rome, Rome, Italy as the disease is frequently locally advanced or metastatic at the time of diagnosis [4]. Even after a successful surgical resection, prognosis is poor. As a matter of fact, in patients undergoing surgery alone the 2-year survival rate is between 30 and $42 \%$, mostly due to the highly frequent recurrences [5]. These results have led to the introduction of multimodal treatments as gold standard in the main international guidelines [6-8]. Adjuvant chemotherapy has been established as an effective strategy to lengthen the diseasefree and overall survival of patients with resectable disease by several clinical trials such as ESPAC-1 [9], ESPAC-3 [10], PRODIGE-24 [11]. However, a significant group of patients is not able to go through any adjuvant treatment: as a matter of fact, it has been reported that $40-53 \%$ of patients do not receive postoperative antineoplastic treatments. This is mostly due to disease progression, surgical complications and poor performance status [12]. Therefore, taking into consideration the importance of a multimodal approach in $\mathrm{PaC}$ and the difficulties in completing the whole treatment plan, neoadjuvant therapy has been seen as a possible treatment option for resectable, borderline resectable and locally advanced PaC. The recent outbreak of the COVID-19 
pandemic has further increased the complexity of balancing the risks and benefits of undergoing preoperative chemotherapy or upfront surgery [13].

The aim of this paper is to offer a state-of-the-art review on neoadjuvant treatments in the setting of pancreatic ductal adenocarcinoma.

\section{Methods}

A systematic literature search was performed by two authors (AC, NP) using PubMed, Cochrane, Web of Science and Embase databases, in order to identify relevant studies published up to and including July 2021 that reported and analyzed the role of neoadjuvant therapy in the setting of pancreatic carcinoma. The search was conducted using the following search algorithm: [(pancreas OR pancreatic AND adenocarcinoma OR carcinoma OR cancer AND neoadjuvant $\mathrm{OR}$ preoperative). The research was restricted to English language articles dealing with human patients. The "related articles" function was used to broaden the search, and all abstracts, studies and citations scanned were reviewed. Relevant papers were selected following the PRISMA statement for systematic reviews and meta-analysis [14]. The risk of bias was evaluated using the Cochrane tool and studies at high risk of bias were excluded. The flowchart of the included studies is shown in Fig. 1.

\section{Potential benefits of neoadjuvant therapy}

As highlighted by a survey performed by E-AHPBA and EORTC in 2019 [15], the reasons behind preoperative treatment in resectable and non-resectable $\mathrm{PaC}$ are different. It might be useful to define preoperative therapy in RPC as "neoadjuvant", as it has the objective of reducing recurrence and $\mathrm{R} 1$ resections in an already resectable tumor. On the other hand, preoperative treatment in case of unresectable tumors aims to increase resection rates, and therefore it has been referred in the mentioned paper as "conversion" or "downsizing" therapy. Such differentiation might be of use when it comes to comparing results in the borderline resectable group. Preoperative chemotherapy is gaining more and more consensus as it allows some patients with locally advanced (LAPC) or borderline resectable (BRPC) pancreatic cancer to access effective surgical treatment $[6$, $16,17]$. The benefits of neoadjuvant therapy have been studied and are starting to gain relevance even in resectable pancreatic cancer (RPC) [18], as it might help increase the number of patients who receive systemic treatment and increase the rate of margin-negative resections. Furthermore, is has been reported that neoadjuvant therapy can select the patients with progressive disease, that therefore might not benefit from surgery. It is also believed that the administration of antineopalstic agents before surgery might be more effective (as free-radicals generation is greater in a well oxygenated environment), associated with less systemic toxicity and protective of intraperitoneal tumor seeding at time of surgery. Furthermore, neoadjuvant treatment may be able to treat micrometastasis that may be present at the time of diagnosis [19]. As a matter of fact, neoadjuvant therapy was associated with a lower rate of both local, lymphnodal and liver recurrences in two retrospective studies [20,21]. Moreover, chemotherapy followed by surgical resection has been studied in the treatment of oligometastatic pancreatic cancer with resectable liver metastases in strictly selected patients; although strong evidence about the potential benefits of this strategy is still lacking $[17,22]$.

Pancreatic surgery remains extremely challenging, with high complication rates, therefore the increase in preoperative therapy administration raised some doubts around the safety of pancreatic resections after neoadjuvant treatments. However, even though increased blood loss and surgical time might occur, overall post-surgical morbidity is not affected [23]. Studies even show a decrease in postoperative pancreatic fistula (POPF) when compared to upfront surgery, although associated with an higher rate of delayed gastric emptying [24, 25]. Nevertheless, among the patients who develop a postoperative complication, those who underwent NAT were associated with increased postoperative burden, measured through the Modified Accordion Severity Grading System and average complication burden (ACB) [26].

\section{How to evaluate the response to NAT}

Preoperative assessment of NAT response is crucial especially in patients with locally advanced and borderline resectable disease, to effectively select patients for surgery. However, a correct disease assessment after NAT is challenging and the best diagnostic option to evaluate patients after neoadjuvant treatment is still not clear. As a matter of fact, even though CT still remains the most commonly used imaging tool to be used to assess disease progression after neoadjuvant therapy, its accuracy in predicting surgical resectability is debated. Ferrone et al. [24] suggested that CT scan is no longer appropriate in assessing resectability after FOLFIRINOX. This result can be explained by the inability of CT to distinguish between fibrosis and neoplasia. 18F-glucose Positron emission tomography has been found to be more accurate of both CT scan and MRI, however, high-quality evidence is still lacking [26, 27].

The best way to evaluate disease response to neoadjuvant therapy is pathological: nevertheless, pathologic response can only be assessed after surgical resection. Complete pathologic response is rare (around 4\%) and often overestimated [28], but associated with significant improvement in surgical outcomes (43 months vs 24 and 


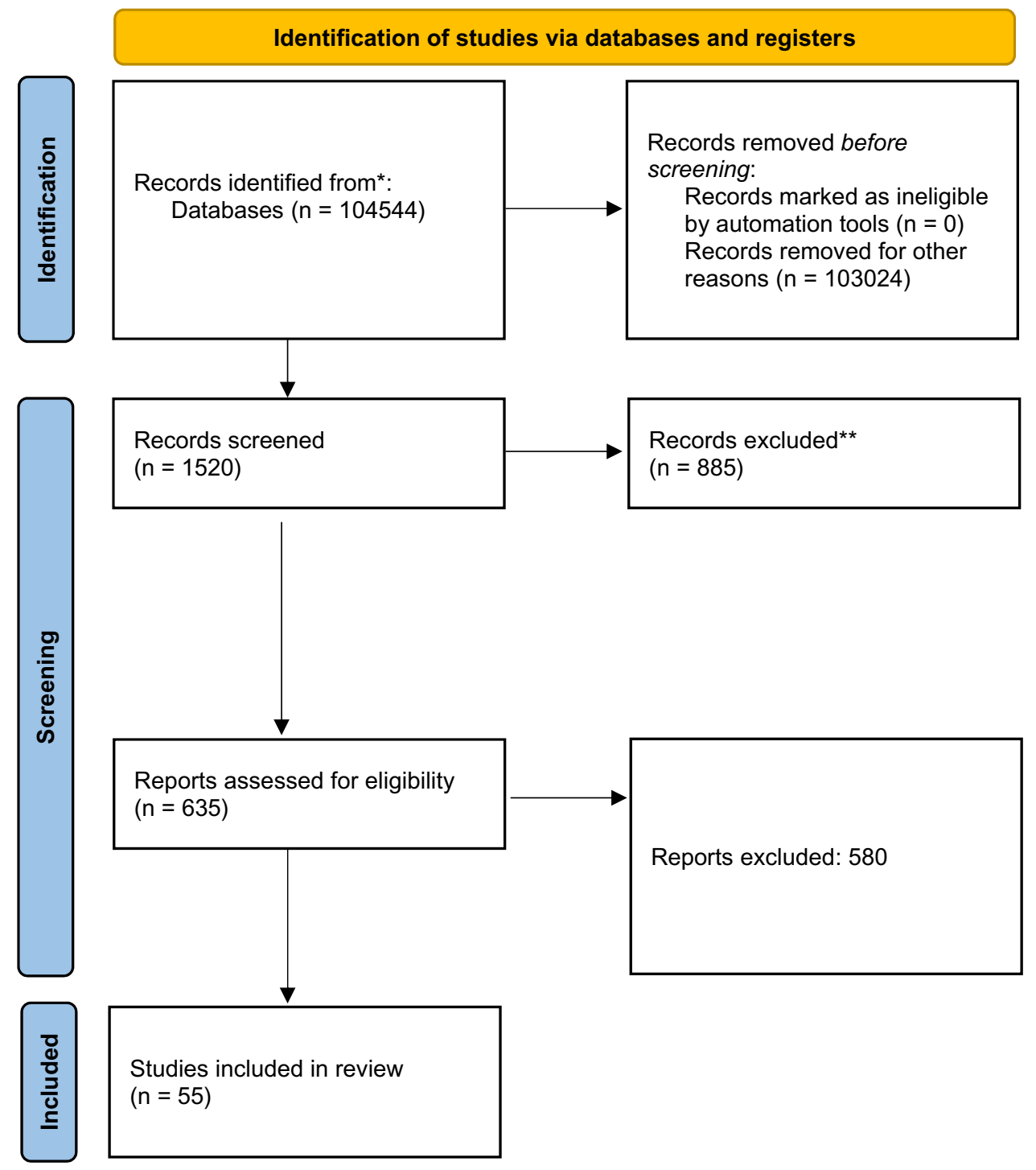

${ }^{*}$ Consider, if feasible to do so, reporting the number of records identified from each database or register searched (rather than the total number across all databases/registers).

** If automation tools were used, indicate how many records were excluded by a human and how many were excluded by automation tools.

Fig. 1 PRISMA 2020 flow diagram for new systematic reviews which included searches of databases and registers only. From: Page MJ, McKenzie JE, Bossuyt PM, Boutron I, Hoffmann TC, Mulrow CD, et al. The PRISMA 2020 statement: an updated guideline for report-

23 months in case of near-complete pathologic response and incomplete response, respectively; $P<0.0001)[29$, 30].

CA19-9 is a serum marker often used in clinical setting for diagnosis and follow-up of pancreatic cancer, and it has been studied as a useful tool to evaluate the response to preoperative treatments [31]. Tsai et al. [32] suggested that the ing systematic reviews. BMJ 2021;372:n71. https://doi.org/10.1136/ bmj.n71. For more information, visit: http://www.prisma-statement. org/

normalization of CA19-9 has a stronger prognostic value than breadth of the reduction.

\section{Treatment regimens}

Regarding the types of regiments to use, current literature is not clear. Combination therapies, especially FOLFIRINOX 
[12], seem to be the most effective as adjuvant treatments, and have shown the most promising results as neoadjuvant therapies in resectable and borderline resectable pancreatic tumors compared to monotherapies [33, 34]. The PACT15 [5] trial showed an increased overall survival after perioperative PEXG over adjuvant gemcitabine or adjuvant PEXG + gemcitabine for RPC, with an ITT 5-year overall survival of $49 \%$ (13\% in patients receiving only gemcitabine; $24 \%$ in patients receiving a combination of PEXG and gemcitabine). The early results from a recent randomized trial (SWOG S1505) confronting neoadjuvant mFOLFIRINOX versus Gemcitabine/Nab-paclitaxel for perioperative treatment in RPC were published: although the comparative results between the two regimens are yet to be published, this trial showed promising outcomes after preoperative chemotherapy, with a $33 \%$ rate of complete or near-complete pathological response; however, it highlighted the challenges of administering complete perioperative therapy [35]. Currently there are no clear guidelines on which neoadjuvant systemic protocol is better to increment resection rates and survival in patients with LAPC. The use of FOLFIRINOX in the setting of metastatic pancreatic cancer has been associated with an increased survival over gemcitabine, at the expense of higher toxicities, deemed, therefore, more appropriate for young and fit patients [36]. However, recent results from the multicenter retrospective RESPECT study found similar oncological outcomes and resection rates between patients $>70$-years old and patients $<70$-years old who underwent treatment with FOLFIRINOX for BRPC or LAPC: this could possibly expand the pool of patients who might benefit from FOLFIRINOX [37].

Even though radiotherapy might help control local disease recurrence, the role of radiotherapy in the preoperative treatment of RPC is still not clear, as, to our knowledge, there are no studies comparing neoadjuvant chemotherapy to radiotherapy or chemoradiotherapy for the treatment of RPC or BRPC. However, Stessin et al. reported the results of their retrospective study using the SEER Registry, that show how neoadjuvant radiotherapy \pm chemotherapy may increase overall survival compared to adjuvant radiotherapy and no radiotherapy [38]. Considering the results of LAP07 trial, no significant difference was found in terms of OS between chemotherapy and chemoradiotherapy in LAPC [39]; therefore chemotherapy alone is still considered as the upfront treatment in LAPC over chemoradiotherapeutic regimens. Recently, Stereotactic Body Radiation Therapy (SBRT) use has been implemented: its advantage compared to standard radiotherapy is the possibility of administering a higher radiation dose selectively on the tumor, sparing surrounding tissues. Given its ability to increase radical surgical resection, SBRT has been inserted in the NCCN guidelines as a feasible option in the setting of LAPC, although more strong evidence is needed. $[6,40]$.

\section{Neoadjuvant therapy in resectable pancreatic cancer}

The NCCN Guidelines still recommend upfront surgery as the first therapeutic choice in most resectable pancreatic cancer cases, limiting NAT only to extremely selected, high-risk patients. However, neoadjuvant treatment is being studied as a therapeutic option to improve the patients' outcomes and to increase resection rate. To our knowledge the only published multicenter randomized controlled trial comparing neoadjuvant chemoradiotherapy to upfront surgery in RPC and BRPC is the 2020 PREOPANC trial [41]; it did not show a significant OS and DFS benefit in the subgroup of patients with resectable disease at time of diagnosis undergoing neoadjuvant chemoradiotherapy (NAT 14.6 months vs immediate surgery 15.6 months; $P=0.830$ ). However, the overall results, comprehensive of both RPC and BRPC, showed a significant increase in DFS and R0 resections after neoadjuvant therapy (DFS: NAT 8.1 months vs immediate surgery 7.7 months; $P=0.032$; R0 rate: NAT $71 \%$ vs immediate surgery $40 \% ; P<0.001$ ); no significant OS difference was found. Of those who received neoadjuvant chemotherapy $17.6 \%$ did not undergo surgery: the most common reason for surgery exclusion was disease progression $(81.25 \%)$. These results are in line with previous single center randomized trials, two of which were terminated early because of poor accrual $[42,43]$. However, there are several limitations to this trial, especially regarding the regiments used: as highlighted before, multi-drug regimens have shown to be the most effective in pancreatic cancer, while in the PREOPANC trial gemcitabine-based monotherapies where used. The study protocol for PREOPANC-2 trial has been recently published, comparing neoadjuvant FOLFIRINOX to neoadjuvant gemcitabine and adjuvant gemcitabine [44].

Data supporting neoadjuvant therapy in RPC still needs to be implemented. However, there are some difficulties in gaining high quality information on this topic. As a matter of fact, there are difficulties in recruiting patients for randomized trials, as they often refuse chemoradiotherapy over the possibility of immediate surgery. Moreover, retrospective studies are often biased: Versteijne et al. [45], indeed, highlight a major selection bias, typical of retrospective studies regarding neoadjuvant therapy. In fact, it is often not clear how many patients with resectable disease at time of diagnosis undergoing neoadjuvant therapy are excluded from surgery because of disease progression or poor PS, as most of the studies report survival after resection rather than by intention-to-treat. This datum, however, is of outmost importance, as one of the major advantages attributed to neoadjuvant therapy in RPC is the possibility to select patients who can gain an advantage from surgery by identifying undetected metastases and the patient's physiologic reserve. As a matter of fact, a retrospective analysis 
performed at the MD Anderson Cancer Center shows that around $25 \%$ of patients have progressive disease at restaging after neoadjuvant therapy and therefore are deemed not resectable [46].

Recently, as cited earlier, Ahmad et al. published the early results of the SWOG S1505 trial [35]: the authors highlighted the fact that the adherence to strict definitions of resectable disease is challenging, and this inaccuracy may lead to a high rate of ineligibility (30\%) in clinical trials.

More randomized control trials are needed to fully understand the implications of neoadjuvant therapy in the setting of RPC. The ongoing PANACHE01 multicenter trial is studying the role of neoadjuvant FOLFIRINOX in RPC [47].

\section{Neoadjuvant therapy in borderline resectable pancreatic cancer}

BRPC is complex clinical entity, as oftentimes the resectability criteria may vary between centers, but can be defined as a tumor at high risk of incomplete surgical resection (R1 or R2) if treated with immediate surgery [48]. The 2020 NCCN guidelines recommend neoadjuvant treatment as the first therapy for borderline pancreatic cancer and do no longer recommend upfront surgery, even if deemed feasible. The European Society of Medical Oncology (ESMO) only recommends preoperative treatment in case of BRPC, suggesting that patients with BRPC are not good candidates for upfront surgery and, indeed, can benefit from medical neoadjuvant treatment [7]. The PREOPANC trial showed significant results favoring NAT in BRPC. As a matter of fact, in the borderline resectable group, OS, DFS and R0 resection rate were significantly increased after NAT (OS: NAT 17.6 months vs immediate surgery 13.2 months; $P=0.029$; DFS: NAT 6.3 months vs immediate surgery 6.2 months; $P=0.013$; R0 rate: NAT $79 \%$ vs immediate surgery $13 \%$; $P<0.001)$. Previous trials results were comparable, showing high resection R0 resection rates in BRPC [49, 50]: 93\% of R0 resection rate was achieved in the ALLIANCE A021101 single-arm trial. The same trial showed a high rate of vascular resections (80\%). However, no differences in surgical morbidity were found between NAT and upfront surgery even after complex vascular reconstructions [51]. As achieving an $\mathrm{R} 0$ resection represents the most important prognostic factor for $\mathrm{PaC}$ [52], the fact that neoadjuvant therapy seems to be able to reduce positive-margin resections is of outmost importance. Versteijne et al. [45] confirmed in a 2018 meta-analysis the increase in $\mathrm{R} 0$ resections in both RPC and BRPC after NAT (RPC: NAT $85.5 \%$ vs immediate surgery $71.4 \%$; BRPC: NAT $88.6 \%$ vs immediate surgery $63.9 \%$ ). The fact that the R0 rate for BRPC is higher than the one of resectable tumors is not entirely clear, but it could be explained by the inhomogeneity in the preoperative assessment of surgical resectability between centers. The ongoing
PRODIGE 44 trial, due to end in 2026, aims to assess the role of neoadjuvant FOLFIRINOX associated with capecitabine based chemoradiotherapy [53].

\section{Conversion therapy in locally advanced pancreatic cancer}

LAPC has historically been considered not deemed for surgical resection given the generally aggressive tumor biology and extreme difficulty achieving a complete resection. In this context the possible therapeutic options are either palliative chemotherapy with best supporting care or chemotherapy and/or radiation with the intent of subsequent surgical resection. Less than $40 \%$ of LAPC patients who undergo neoadjuvant treatment eventually become resectable [54]; however, the possibility of surgical resection gives survival advantage over palliative care in selected patients as reported by Gurusamy et al. in a 2014 meta-analysis of two randomized trials [55]. NCCN guidelines recommend surgical treatment after NAT in patients with LAPC with a good performance status (defined as ECOG 0-1) and no signs of disease progression. As high-quality evidence supporting surgical resection after NAT over systemic therapy in LAPC is still scarce, in the clinical setting it is fundamental to carefully select the patients to refer to surgery.

\section{Conclusions}

NAT has an important role in the multimodal treatment of patients with borderline resectable pancreatic cancer. It has a role in patients with locally advanced tumors as it can allow surgical resection in a relevant proportion of patients. For resectable pancreatic cancers, the role of NAT is under evaluation by several randomized trials.

Author contributions JG has given substantial contributions to conception and design and analysis and interpretation of data. NP and AC have made substantial contributions acquisition of data, and analysis and interpretation of data. JG, AC, NP have been involved in drafting the manuscript or revising it critically for important intellectual content. $\mathrm{JG}, \mathrm{AC}$, NP have given final approval of the version to be published.

Funding No funding was received for the present article.

Data availability Not applicable.

Code availability Not applicable.

\section{Declarations}

Conflict of interest The authors JG, AC, NP have no conflicts of interests to declare. 
Ethical approval Not applicable.

Consent to participate Not applicable.

Consent for publication Not applicable.

\section{References}

1. Siegel RL, Miller KD, Jemal A (2020) Cancer statistics, 2020. CA Cancer J Clin 70:7-30. https://doi.org/10.3322/caac.21590

2. Rahib L, Smith BD, Aizenberg R, Rosenzweig AB, Fleshman JM, Matrisian LM (2014) Projecting cancer incidence and deaths to 2030: the unexpected burden of thyroid, liver, and pancreas cancers in the United States. Cancer Res 1(74):2913-2921. https:// doi.org/10.1158/0008-5472.CAN-14-0155

3. Carrato A, Falcone A, Ducreux M, Valle JW, Parnaby A, Djazouli $\mathrm{K}$ et al (2015) A systematic review of the burden of pancreatic cancer in Europe: real-world impact on survival, quality of life and costs. J Gastrointest Cancer 46:201-211. https://doi.org/10.1007/ s12029-015-9724-1

4. Li D, Xie K, Wolff R, Abbruzzese JL (2004) Pancreatic cancer. The Lancet 363:1049-1057. https://doi.org/10.1016/S01406736(04)15841-8

5. Reni M, Balzano G, Zanon S, Zerbi A, Rimassa L, Castoldi R et al (2018) Safety and efficacy of preoperative or postoperative chemotherapy for resectable pancreatic adenocarcinoma (PACT15): a randomised, open-label, phase 2-3 trial. Lancet Gastroenterol Hepatol 3:413-423. https://doi.org/10.1016/S2468-1253(18) 30081-5

6. NCCN guidelines on pancreatic cancer (2020) Available from: https://www.nccn.org/professionals/physician_gls/pdf/pancreatic blocks.pdf. Accessed October 11, 2020

7. Ducreux M, Cuhna AS, Caramella C, Hollebecque A, Burtin P, Goéré D et al (2015) Cancer of the pancreas: ESMO clinical practice guidelines for diagnosis, treatment and follow-up. Ann Oncol 1(26):v56-68. https://doi.org/10.1093/annonc/mdv295

8. Pancreatic cancer in adults: diagnosis and management (2018). National Guideline Alliance (UK) London: National Institute for Health and Care Excellence (UK): Clinical Guidelines. Available from: https://www.ncbi.nlm.nih.gov/books/NBK487233. Accessed October 11, 2020

9. Neoptolemos JP, Stocken DD, Friess H, Bassi C, Dunn JA, Hickey $\mathrm{H}$ et al (2004) A randomized trial of chemoradiotherapy and chemotherapy after resection of pancreatic cancer. N Engl J Med 18(350):1200-1210. https://doi.org/10.1056/NEJMoa032295

10. Neoptolemos JP, Stocken DD, Bassi C, Ghaneh P, Cunningham D, Goldstein D et al (2010) Adjuvant chemotherapy with fluorouracil plus folinic acid vs gemcitabine following pancreatic cancer resection: a randomized controlled trial. JAMA 304:1073-1081. https://doi.org/10.1001/jama.2010.1275

11. Conroy T, Hammel P, Hebbar M, Ben Abdelghani M, Wei AC, Raoul J-L et al (2018) FOLFIRINOX or gemcitabine as adjuvant therapy for pancreatic cancer. N Engl J Med 20(379):2395-2406. https://doi.org/10.1056/NEJMoa1809775

12. Tzeng C-WD, Tran Cao HS, Lee JE, Pisters PWT, Varadhachary GR, Wolff RA et al (2014) Treatment sequencing for resectable pancreatic cancer: influence of early metastases and surgical complications on multimodality therapy completion and survival. J Gastrointest Surg Off J Soc Surg Aliment Tract 18:16-24. https:// doi.org/10.1007/s11605-013-2412-1 (discussion 24-25)

13. Jones CM, Radhakrishna G, Aitken K, Bridgewater J, Corrie $\mathrm{P}$, Eatock M et al (2020) Considerations for the treatment of pancreatic cancer during the COVID-19 pandemic: the UK consensus position. Br J Cancer 8:1-5. https://doi.org/10.1038/ s41416-020-0980-x

14. Liberati A, Altman DG, Tetzlaff J, Mulrow C, Gøtzsche PC, Ioannidis JPA et al (2009) The PRISMA statement for reporting systematic reviews and meta-analyses of studies that evaluate health care interventions: explanation and elaboration. PLoS Med 6(7):e1000100

15. Heinrich S, Besselink M, Moehler M, van Laethem J-L, Ducreux M, Grimminger P et al (2019) Opinions and use of neoadjuvant therapy for resectable, borderline resectable, and locally advanced pancreatic cancer: international survey and case-vignette study. BMC Cancer 19:675. https://doi.org/10. $1186 / \mathrm{s} 12885-019-5889-5$

16. Heinemann V, Haas M, Boeck S (2013) Neoadjuvant treatment of borderline resectable and non-resectable pancreatic cancer. Ann Oncol 24:2484-2492. https://doi.org/10.1093/annonc/mdt239

17. Crippa S, Bittoni A, Sebastiani E, Partelli S, Zanon S, Lanese A et al (2016) Is there a role for surgical resection in patients with pancreatic cancer with liver metastases responding to chemotherapy? Eur J Surg Oncol 42:1533-1539. https://doi.org/10.1016/j. ejso.2016.06.398

18. Russo S, Ammori J, Eads J, Dorth J (2016) The role of neoadjuvant therapy in pancreatic cancer: a review. Future Oncol 12:669685. https://doi.org/10.2217/fon. 15.335

19. Papavasiliou P, Hoffman JP, Cohen SJ, Meyer JE, Watson JC, Chun YS (2014) Impact of preoperative therapy on patterns of recurrence in pancreatic cancer. HPB 16:34-39. https://doi.org/ 10.1111/hpb.12058

20. Sugimoto M, Takahashi N, Farnell MB, Smyrk TC, Truty MJ, Nagorney DM et al (2019) Survival benefit of neoadjuvant therapy in patients with non-metastatic pancreatic ductal adenocarcinoma: a propensity matching and intention-to-treat analysis. J Surg Oncol 120:976-984. https://doi.org/10.1002/jso.25681

21. Christians KK, Heimler JW, George B, Ritch PS, Erickson BA, Johnston F et al (2016) Survival of patients with resectable pancreatic cancer who received neoadjuvant therapy. Surgery 159:893-900. https://doi.org/10.1016/j.surg.2015.09.018

22. Zhou W, Wang D, Lou W (2020) Current role of surgery in pancreatic cancer with synchronous liver metastasis. Cancer Control 27(1):1073274820976593. https://doi.org/10.1177/1073274820 976593 (PMID: 33238715; PMCID: PMC7791445)

23. Nipp RD, Zanconato A, Zheng H, Ferrone CR, Lillemoe KD, Wo JY et al (2017) Predictors of early mortality after surgical resection of pancreatic adenocarcinoma in the era of neoadjuvant treatment. Pancreas 46:183-189. https://doi.org/10.1097/MPA. 0000000000000731

24. Ferrone CR, Marchegiani G, Hong TS, Ryan DP, Deshpande V, McDonnell EI et al (2015) Radiological and surgical implications of neoadjuvant treatment with FOLFIRINOX for locally advanced and borderline resectable pancreatic cancer. Ann Surg 261:12-17. https://doi.org/10.1097/SLA.0000000000000867

25. Marchegiani G, Andrianello S, Nessi C, Sandini M, Maggino L, Malleo G et al (2018) Neoadjuvant therapy versus upfront resection for pancreatic cancer: the actual spectrum and clinical burden of postoperative complications. Ann Surg Oncol 25:626-637. https://doi.org/10.1245/s10434-017-6281-9

26. Yokose T, Kitago M, Matsusaka Y, Masugi Y, Shinoda M, Yagi $\mathrm{H}$ et al (2020) Usefulness of 18F-fluorodeoxyglucose positron emission tomography/computed tomography for predicting the prognosis and treatment response of neoadjuvant therapy for pancreatic ductal adenocarcinoma. Cancer Med 9:4059-4068. https:// doi.org/10.1002/cam4.3044

27. Zimmermann C, Distler M, Jentsch C, Blum S, Folprecht G, Zöphel K et al (2020) Evaluation of response using FDG-PET/ $\mathrm{CT}$ and diffusion weighted MRI after radiochemotherapy of pancreatic cancer: a non-randomized, monocentric phase II clinical 
trial-PaCa-DD-041 (Eudra-CT 2009-011968-11). Strahlenther Onkol. https://doi.org/10.1007/s00066-020-01654-4

28. Laura A, Anna C, Cinquepalmi M, Giovanni M, Sole MM, Nava AK et al (2020) Is complete pathologic response in pancreatic cancer overestimated? A systematic review of prospective studies. J Gastrointest Surg 24:2336-2348. https://doi.org/10.1007/ s11605-020-04697-1

29. Sell NM, Lee GC, Fernández-Del Castillo C, Ferrone CR, Warshaw AL, Hong TS et al (2020) Evaluation of pathologic response on overall survival after neoadjuvant therapy in pancreatic ductal adenocarcinoma. Pancreas 49:897-903. https://doi.org/10.1097/ MPA.0000000000001590

30. He J, Blair AB, Groot VP, Javed AA, Burkhart RA, Gemenetzis $\mathrm{G}$ et al (2018) Is a pathological complete response following neoadjuvant chemoradiation associated with prolonged survival in patients with pancreatic cancer? Ann Surg 268:1-8. https://doi. org/10.1097/SLA.0000000000002672

31. Perri G, Prakash LR, Katz MHG (2020) Response to preoperative therapy in localized pancreatic cancer. Front Oncol 10:516. https://doi.org/10.3389/fonc.2020.00516

32. Tsai S, George B, Wittmann D, Ritch PS, Krepline AN, Aldakkak $\mathrm{M}$ et al (2020) Importance of normalization of CA19-9 levels following neoadjuvant therapy in patients with localized pancreatic cancer. Ann Surg 271:740-747. https://doi.org/10.1097/SLA. 0000000000003049

33. Palmer DH, Stocken DD, Hewitt H, Markham CE, Hassan AB, Johnson PJ et al (2007) A randomized phase 2 trial of neoadjuvant chemotherapy in resectable pancreatic cancer: gemcitabine alone versus gemcitabine combined with cisplatin. Ann Surg Oncol 14:2088-2096. https://doi.org/10.1245/s10434-007-9384-x

34. Christians KK, Tsai S, Mahmoud A, Ritch P, Thomas JP, Wiebe L et al (2014) Neoadjuvant FOLFIRINOX for borderline resectable pancreas cancer: a new treatment paradigm? Oncologist 19:266274. https://doi.org/10.1634/theoncologist.2013-0273

35. Ahmad SA, Duong M, Sohal DPS, Gandhi NS, Beg MS, WangGillam A et al (2020) Surgical outcome results from SWOG S1505: a randomized clinical trial of mFOLFIRINOX versus gemcitabine/nab-paclitaxel for perioperative treatment of resectable pancreatic ductal adenocarcinoma. Ann Surg. https://doi.org/ 10.1097/SLA.0000000000004155

36. Conroy T, Desseigne F, Ychou M, Bouché O, Guimbaud R, Bécouarn Y et al (2011) FOLFIRINOX versus gemcitabine for metastatic pancreatic cancer. N Engl J Med 364:1817-1825. https:// doi.org/10.1056/NEJMoa1011923

37. Weniger M, Moir J, Damm M, Maggino L, Kordes M, Rosendahl J et al (2020) Neoadjuvant therapy in elderly patients receiving FOLFIRINOX or gemcitabine/nab-paclitaxel for borderline resectable or locally advanced pancreatic cancer is feasible and lead to a similar oncological outcome compared to non-aged patients-results of the RESPECT-Study. Surg Oncol 35:285297. https://doi.org/10.1016/j.suronc.2020.08.031

38. Stessin AM, Meyer JE (2008) Sherr DL (2008) Neoadjuvant radiation is associated with improved survival in patients with resectable pancreatic cancer: an analysis of data from the surveillance, epidemiology, and end results (SEER) registry. Int J Radiat Oncol Biol Phys 72:1128-1133. https://doi.org/10.1016/j.ijrobp.2008.02. 065

39. Hammel P, Huguet F, van Laethem J-L, Goldstein D, Glimelius B, Artru P et al (2016) Effect of chemoradiotherapy vs chemotherapy on survival in patients with locally advanced pancreatic cancer controlled after 4 months of gemcitabine with or without erlotinib: the lap07 randomized clinical trial. JAMA 315:1844-1853. https://doi.org/10.1001/jama.2016.4324

40. Moningi S, Dholakia AS, Raman SP, Blackford A, Cameron JL, Le DT, De Jesus-Acosta AM, Hacker-Prietz A, Rosati LM, Assadi RK, Dipasquale S, Pawlik TM, Zheng L, Weiss MJ, Laheru DA,
Wolfgang CL, Herman JM (2015) The role of stereotactic body radiation therapy for pancreatic cancer: a single-institution experience. Ann Surg Oncol 22(7):2352-2358. https://doi.org/10. 1245/s10434-014-4274-5 (Epub 2015 Jan 7. PMID: 25564157; PMCID: PMC4459890)

41. Versteijne E, Suker M, Groothuis K, Akkermans-Vogelaar JM, Besselink MG, Bonsing BA et al (2020) Preoperative chemoradiotherapy versus immediate surgery for resectable and borderline resectable pancreatic cancer: results of the Dutch randomized phase III PREOPANC trial. J Clin Oncol 38:1763-1773. https:// doi.org/10.1200/JCO.19.02274

42. Casadei R, Di Marco M, Ricci C, Santini D, Serra C, Calculli L et al (2015) Neoadjuvant chemoradiotherapy and surgery versus surgery alone in resectable pancreatic cancer: a single-center prospective, randomized, controlled trial which failed to achieve accrual targets. J Gastrointest Surg 19:1802-1812. https://doi.org/ 10.1007/s11605-015-2890-4

43. Golcher H, Brunner TB, Witzigmann H, Marti L, Bechstein W-O, Bruns $C$ et al (2015) Neoadjuvant chemoradiation therapy with gemcitabine/cisplatin and surgery versus immediate surgery in resectable pancreatic cancer: results of the first prospective randomized phase II trial. Strahlenther Onkol 191:7-16. https://doi. org/10.1007/s00066-014-0737-7

44. Janssen QP, van Dam JL, Bonsing BA, Bos H, Bosscha KP, Coene PPLO, van Eijck CHJ, de Hingh IHJT, Karsten TM, van der Kolk MB, Patijn GA, Liem MSL, van Santvoort HC, Loosveld OJL, de Vos-Geelen J, Zonderhuis BM, Homs MYV, van Tienhoven G, Besselink MG, Wilmink JW, Groot KB, Dutch Pancreatic Cancer Group (2021) Total neoadjuvant FOLFIRINOX versus neoadjuvant gemcitabine-based chemoradiotherapy and adjuvant gemcitabine for resectable and borderline resectable pancreatic cancer (PREOPANC-2 trial): study protocol for a nationwide multicenter randomized controlled trial. BMC Cancer 21(1):300. https://doi. org/10.1186/s12885-021-08031-z (PMID: 33757440; PMCID: PMC7989075)

45. Versteijne E, Vogel JA, Besselink MG, Busch ORC, Wilmink JW, Daams JG et al (2018) Meta-analysis comparing upfront surgery with neoadjuvant treatment in patients with resectable or borderline resectable pancreatic cancer. Br J Surg 105:946-958. https:// doi.org/10.1002/bjs. 10870

46. Breslin TM, Hess KR, Harbison DB, Jean ME, Cleary KR, Dackiw AP et al (2001) Neoadjuvant chemoradiotherapy for adenocarcinoma of the pancreas: treatment variables and survival duration. Ann Surg Oncol 8:123-132. https://doi.org/10.1007/ s10434-001-0123-4

47. Schwarz L, Vernerey D, Bachet J-B, Tuech J-J, Portales F, Michel $P$ et al (2018) Resectable pancreatic adenocarcinoma neo-adjuvant FOLF(IRIN)OX-based chemotherapy-a multicenter, noncomparative, randomized, phase II trial (PANACHE01-PRODIGE48 study). BMC Cancer 18:762. https://doi.org/10.1186/ s12885-018-4663-4

48. Isaji S, Mizuno S, Windsor JA, Bassi C, Fernández-del Castillo C, Hackert $\mathrm{T}$ et al (2018) International consensus on definition and criteria of borderline resectable pancreatic ductal adenocarcinoma 2017. Pancreatology 18:2-11. https://doi.org/10.1016/j.pan.2017. 11.011

49. Esnaola NF, Chaudhary UB, O'Brien P, Garrett-Mayer E, Camp ER, Thomas MB et al (2014) Phase 2 trial of induction gemcitabine, oxaliplatin, and cetuximab followed by selective capecitabine-based chemoradiation in patients with borderline resectable or unresectable locally advanced pancreatic cancer. Int J Radiat Oncol Biol Phys 88:837-844. https://doi.org/10.1016/j.ijrobp. 2013.12.030

50. Katz MHG, Shi Q, Ahmad SA, Herman JM, de MarshCollisson RWE et al (2016) Preoperative modified FOLFIRINOX treatment followed by capecitabine-based chemoradiation for borderline 
resectable pancreatic cancer: alliance for clinical trials in oncology trial A021101. JAMA Surg 151:e161137. https://doi.org/10. 1001/jamasurg.2016.1137

51. Peters NA, Javed AA, Cameron JL, Makary MA, Hirose K, Pawlik TM et al (2016) Modified Appleby procedure for pancreatic adenocarcinoma: does improved neoadjuvant therapy warrant such an aggressive approach? Ann Surg Oncol 23:3757-3764. https://doi.org/10.1245/s10434-016-5303-3

52. Ren W, Xourafas D, Ashley SW, Clancy TE (2020) Temporal assessment of prognostic factors in patients with pancreatic ductal adenocarcinoma undergoing neoadjuvant treatment and resection. J Surg Res 257:605-615. https://doi.org/10.1016/j.jss.2020.07.073

53. Institut de Cancérologie de Lorraine. Two Arm, Prospective, Multicenter Randomized Phase II Trial of Neoadjuvant Modified Folfirinox Regimen, With or Without Preoperative Concomitant Chemoradiotherapy in Patients With Borderline Resectable Pancreatic Carcinoma (2020). clinicaltrials.gov. Report No.:
NCT02676349. Available from: https://clinicaltrials.gov/ct2/ show/NCT02676349. Accessed October 11, 2020

54. Kluger MD, Rashid MF, Rosario VL, Schrope BA, Steinman JA, Hecht EM et al (2018) Resection of locally advanced pancreatic cancer without regression of arterial encasement after modern-era neoadjuvant therapy. J Gastrointest Surg 22:235-241. https://doi. org/10.1007/s11605-017-3556-1

55. Gurusamy KS, Kumar S, Davidson BR, Fusai G (2014) Resection versus other treatments for locally advanced pancreatic cancer. Cochrane Database Syst Rev 2:CD010244. https://doi.org/10. 1002/14651858.CD010244.pub2

Publisher's Note Springer Nature remains neutral with regard to jurisdictional claims in published maps and institutional affiliations. 